VOL. $70(2004) \quad$ [377-383]

\title{
POINTED BLASCHKE MANIFOLDS AND GEODESIC NORMAL SECTIONS
}

\author{
Dong-Soo Kim, Young Ho Kim and Eun Kyoung Lee
}

\begin{abstract}
We study complete submanifolds of Euclidean space where every geodesic passing through a fixed point is the normal section along it. We prove that all such geodesics are independent of the direction at the point and such submanifolds are pointed Blaschke manifolds or diffeomorphic to a Euclidean space.
\end{abstract}

\section{INTRODUCTION}

It is interesting to study Riemannian manifolds by examining the behaviour of their geodesics. When a geodesic of a submanifold $M$ of a Riemannian manifold $\widetilde{M}$ is viewed as a curve in $\widetilde{M}$, we may use the theory of submanifolds. A submanifold $M$ in a Riemannian manifold $\widetilde{M}$ is called planar geodesic if every geodesic of $M$ is contained in a 2-dimensional totally geodesic subamniofold of $\widetilde{M}$ and helical if every geodesic of $M$ regarded as a curve of $\widetilde{M}$ has constant Frenet curvatures independent of the choice of geodesic $([\mathbf{1}, \mathbf{5}, \mathbf{9}, 1 \mathbf{1}$, $12,13,14]$ ). As a matter of fact, a helical submanifold in a space form is classified as one of compact rank one symmetric spaces or a Euclidean space $([12,13])$. Also, Chen and Verheyen studied submanifolds of Euclidean space with geodesic normal sections. They proved such submanifolds are in fact helical ([3]) and later Verheyen showed that the converse is also true ([15]). It is well known that helical submanifolds of Euclidean space are isometric to either a Blaschke manifold or a manifold diffeomorphic to a Euclidean space $([\mathbf{1 2}])$.

On the other hand, one of the authors studied the so-called pointed helical submanifolds of Euclidean which admit a point $o$ such that all the geodesics passing through $o$ have the same constant curvatures $([6,7,8])$. Recently, he proved that the pointed planar geodesic submanifolds of Euclidean space are geodesically symmetric with respect to a point $([8])$. In 1998, Fueki $([4])$ extended the notion of pointed helical submanifolds of Euclidean space to the pseudo-Riemannian version of it.

In the present paper we study a submanifold $M$ of Euclidean space $\mathbb{E}^{m}$, which admits a fixed point $o$ of $M$ such that every geodesic $\gamma$ of $M$ passing through $o$ is a normal section

Received 10th May, 2004

The second author was supported by Korea Research Foundation (KRF-2003-C00059).

Copyright Clearance Centre, Inc. Serial-fee code: 0004-9727/04 \$A2.00+0.00. 
along $\gamma$, which will be called pointed geodesic normal sections. We prove such a manifold is a Blaschke manifold at $o$ or a manifold diffeomorphic to a Euclidean space.

Therefore, the so-called submanifolds with geodesic normal sections in a Euclidean space or the helical submanifolds in a Euclidean space are obtained from this notion of pointed geodesic normal sections $([3,12])$ and we have a simple and direct classification theorem for those submanifolds.

\section{Preliminaries}

Let $x: M \rightarrow \mathbb{E}^{m}$ be an isometric immersion of an $n$-dimensional Riemannian manifold $M$ into a Euclidean $m$-space $\mathbb{E}^{m}$. Let $\langle\cdot, \cdot\rangle$ be the standard Euclidean metric tensor of $\mathbb{E}^{m}$. Then $M$ has the induced metric from that of $\mathbb{E}^{m}$ that is denoted by the same notation $\langle\cdot, \cdot\rangle$ unless we have confusion. We denote by $\widetilde{\nabla}$ the Levi-Civita connection on $\mathbb{E}^{m}$ and $\nabla$ the induced connection on $M$. Then, we have the Gauss equation $\widetilde{\nabla}_{X} Y=\nabla_{X} Y+h(X, Y)$, where $X$ and $Y$ denote vector fields on $M$ and $h$ is the second fundamental form. The equation of Weingarten is also given by $\widetilde{\nabla}_{X} \xi=-A_{\xi} X+\nabla_{X}^{\perp} \xi$, where $A_{\xi}$ is the Weingarten map associated with a normal vector field $\xi$ to $M$ and $\nabla^{\perp}$ the normal connection in the normal bundle $T^{\perp} M$. As is well known, the Weingarten map $A_{\xi}$ and the second fundamental form $h$ are related by $\left\langle A_{\xi} X, Y\right\rangle=\langle h(X, Y), \xi\rangle$ for all vector fields $X$ and $Y$ on $M$ and $\xi$ normal to $M$. We now define the covariant derivative of $h$ on the direct sum of the tangent bundle and the normal bundle $T M \oplus T^{\perp} M$ of $M$ as

$$
\left(\bar{\nabla}_{X} h\right)(Y, Z)=\nabla_{X}^{\perp} h(Y, Z)-h\left(\nabla_{X} Y, Z\right)-h\left(Y, \nabla_{X} Z\right)
$$

for all vector fields $X, Y$ and $Z$ on $M([2])$. We denote $\left(\bar{\nabla}_{X} h\right)(Y, Z)$ by $(\bar{\nabla} h)(X, Y, Z)$ which is a tensor field of type $(1,3)$. We can also define the higher order covariant derivatives of the second fundamental form $h$ : For a positive integer $k$, we define

$$
\begin{aligned}
\left(\bar{\nabla}^{k} h\right)\left(X_{1}, X_{2}, \ldots, X_{k+2}\right)=\nabla_{X_{1}}^{\frac{1}{1}}\left(\left(\bar{\nabla}^{k-1} h\right)\right. & \left.\left(X_{2}, \ldots, X_{k+2}\right)\right) \\
& -\sum_{i=2}^{k+2}\left(\bar{\nabla}^{k} h\right)\left(X_{2}, X_{3}, \ldots, \nabla_{X_{1}} X_{i}, \ldots, X_{k+2}\right) .
\end{aligned}
$$

We simply denote $\left(\bar{\nabla}^{k} h\right)(X, X, \ldots, X)=\left(\bar{\nabla}^{k} h\right)\left(X^{k+2}\right)$ and $\left(\bar{\nabla}^{0} h\right)(X, X)=h(X, X)$ for any vector field $X$ on $M$.

Let $R$ be the curvature tensor of $M$. The Gauss equation is then given by

$$
\langle R(X, Y) Z, W\rangle=\langle h(X, W), h(Y, Z)\rangle-\langle h(Y, W), h(X, Z)\rangle
$$

for vector fields $X, Y, Z$ and $W$ on $M$. We also obtain the Codazzi equation $(\bar{\nabla} h)(X, Y, Z)-(\bar{\nabla} h)(Y, X, Z)=0$ for all vector fields $X, Y, Z$ on $M$. The submanifold $M$ in a Euclidean space $\mathbb{E}^{m}$ is said to be isotropic at $p \in M$ if the normal curvature of curves 
passing through $p$ is independent of the choice of the curve, that is, $\langle h(t, t), h(t, t)\rangle$ does not depend on the choice of the unit vector $t$ tangent to $M$ at $p$. By O'Neill ([10]), $M$ is isotropic at $p$ if and only if $\left\langle h(t, t), h\left(t, t^{\perp}\right)\right\rangle=0$ for all unit vectors $t$ and $t^{\perp}$ perpendicular to $t$.

For a point $p \in M$ and a unit vector $t$ tangent to $M$ at $p$, the vector $t$ and the normal space $T_{p}^{\perp} M$ of $M$ at $p$ form an $(m-n+1)$-dimensional affine space $E(p ; t)$ in $\mathbb{E}^{m}$ through $p$. The intersection of $M$ with $E(p ; t)$ gives rise to a curve in a neighbourhood of $p$ which is called the normal section at $p$ in the direction $t$. A submanifold $M$ in a Euclidean space $\mathbb{E}^{m}$ is said to have geodesic normal sections if all the geodesics of $M$ are normal sections ([3]).

A submanifold $M$ of a Euclidean space $\mathbb{E}^{m}$ is called pointed planar geodesic at a point $o$ if every geodesic of $M$ through $o$ is a plane curve regarded as a curve in $\mathbb{E}^{m}$.

We now introduce the Frenet curvatures and the Frenet frame of a unit speed curve $\gamma: I \rightarrow \mathbb{E}^{m}$. where $I$ is an open interval. Let $\gamma^{\prime}(s)=T_{1}(s)$ and put $\kappa_{1}=\left\|\widetilde{\nabla}_{T_{1}} T_{1}\right\|$. If $\kappa_{1}=\left\|\widetilde{\nabla}_{T_{1}} T_{1}\right\|$ is identically zero on $I$, then $\gamma$ is said to be of order 1. If $\kappa_{1}=\left\|\tilde{\nabla}_{T_{1}} T_{1}\right\|$ is not identically zero, then one can define a unit vector field $T_{2}$ by $\tilde{\nabla}_{T_{1}} T_{1}=\kappa_{1} T_{2}$ on $I_{1}=\left\{s \in I \mid \kappa_{1}(s) \neq 0\right\}$. Set $\kappa_{2}=\left\|\widetilde{\nabla}_{T_{1}} T_{2}+\kappa_{1} T_{1}\right\|$. If $\kappa_{2}$ is identically zero on $I_{1}$, then $\gamma$ is said to be of order 2. Inductively, we can define $T_{d}$ and $\kappa_{d}=\left\|\widetilde{\nabla}_{T_{1}} T_{d}+\kappa_{d-1} T_{d-1}\right\|$ and if $\kappa_{d}=0$ identically on $I_{d-1}=\left\{s \in I \mid \kappa_{d-1} \neq 0\right\}$, then $\gamma$ is said to be of order $d$. If $\gamma$ is of order $d$, then we have a matrix equation $\tilde{\nabla}_{T_{1}}\left(T_{1}, T_{2}, \ldots, T_{d}\right)=\left(T_{1}, T_{2}, \ldots, T_{d}\right) \Lambda$ on $I_{d-1}$, where $\Lambda$ is a $d \times d$-matrix given by

$$
\Lambda=\left(\begin{array}{cccccc}
0 & -\kappa_{1} & 0 & \ldots & \ldots & 0 \\
\kappa_{1} & 0 & -\kappa_{2} & 0 & \ldots & 0 \\
0 & \kappa_{2} & 0 & \ldots & \ldots & 0 \\
\ldots & & \ldots & \ldots & & -\kappa_{d-1} \\
0 & 0 & \ldots & & \kappa_{d-1} & 0
\end{array}\right) .
$$

The matrix $\Lambda,\left\{T_{1}, T_{2}, \ldots, T_{d}\right\}$ and $\kappa_{1}, \ldots, \kappa_{d-1}$ are called the Frenet formula, the Frenet frame and the Frenet curvatures of $\gamma$ respectively.

We also call $M$ pointed helical at $o$ if every geodesic through $o$ has constant Frenet curvatures that are independent of the choice of geodesic through $o([\mathbf{7}, 8])$. By a simple curve $\gamma$ in a Euclidean space, we mean all of the Frenet curvatures of $\gamma$ are constant along $\gamma([1])$.

Next, we consider the notion of Blaschke manifold. Let $\widetilde{M}$ be a complete Riemannian manifold and $p$ and $q$ points of $\widetilde{M}$. We denote by $U_{p} \widetilde{M}$ the unit tangent space of $\widetilde{M}$ at the point $p$. Then, the link $\Lambda(p, q)$ from $p$ to $q$ is defined as

$$
\Lambda(p, q)=\left\{\gamma^{\prime}(q) \in U_{q} \widetilde{M} \mid \gamma \in \operatorname{Seg}(p, q)\right\},
$$

where $\gamma$ is assumed to be parametrised by the arc length and $\operatorname{Seg}(p, q)$ denotes the set of minimal geodesics joining $p$ to $q$. A compact Riemannian manifold $\widetilde{M}$ is called a 
Blaschke manifold at $p$ if for every point $q$ in $\operatorname{Cut}(p)$ the link $\Lambda(p, q)$ is a great sphere of $U_{q} \widetilde{M}$, where $\operatorname{Cut}(p)$ denotes the cut locus of $p$. The manifold $\widetilde{M}$ is said to be a Blaschke manifold if it is a Blaschke manifold at every point of $\widetilde{M}$. A Blaschke manifold at a point $p$ is characterised by the spherical cut locus at $p$, that is, the cut value is independent of the choice of geodesic emanating from $p$ (see [1]).

\section{Submanifolds with Pointed Geodesic Normal Sections}

Let $M$ be an $n$-dimensional submanifold of an $m$-dimensional Euclidean space $\mathbb{E}^{m}$. A submanifold $M$ in a Euclidean space is said to have pointed geodesic normal sections if there is a point $o$ in $M$ through which all the geodesics are the normal sections along each of geodesics.

Let $\gamma$ be a geodesic of $M$ passing through $o$. Without loss of generality, we may assume $\gamma$ is parametrised by the arc length $s$. From now on, all the geodesics through $o$ are assumed to be parametrised by the arc length unless stated otherwise.

Proposition 3.1. Let $M$ be an n-dimensional submanifold of an $m$ dimensional Euclidean space $\mathbb{E}^{m}$ with pointed geodesic normal sections at $o$. Then, $M$ is isotropic at $o$.

Proof: Choose a geodesic $\gamma$ passing through $o$. Let $\gamma(0)=0$ and $\gamma^{\prime}(s)$ $=T(s)$. Since $\gamma$ is a normal section along the geodesic $\gamma$, the tangential part of $\gamma^{(k)}(s)$ is proportional to $\gamma^{\prime}(s)$ for every $s$ and every positive integer $k$, where $\gamma^{(k)}$ denotes the $k$-th derivative of $\gamma$ in $\mathbb{E}^{m}$. Thus, $\gamma^{\prime \prime \prime}(0)=-A_{h(t, t)} t+(\bar{\nabla} h)\left(t^{3}\right)$ yields $A_{h(t, t)} t \wedge t=0$, where $t=T(0)$ and $(\bar{\nabla} h)\left(t^{3}\right)=(\bar{\nabla} h)(t, t, t)$. It follows that

$$
\left\langle h(t, t), h\left(t, t^{\perp}\right)\right\rangle=0
$$

for all $t^{\perp}$ orthogonal to $t$. Therefore, $M$ is isotropic at $o$.

In order to prove our main theorem, we need the following two lemmas.

LEMma 3.2. Let $M$ be a submanifold of a Euclidean space $\mathbb{E}^{m}$ with pointed geodesic normal sections at $o$. Let $\gamma$ be a geodesic of $M$ passing through $o$. Then, $\left\langle\left(\bar{\nabla}^{(k-2)} h\right)\left(T^{k}\right), h\left(T, T^{\perp}\right)\right\rangle=0$ and $\left\langle\left(\bar{\nabla}^{(k-2)} h\right)\left(T^{\perp}, T^{k-1}\right), h(T, T)\right\rangle=0$ hold, where $T(s)=\gamma^{\prime}(s), T^{\perp}$ is a vector field along $\gamma$ orthogonal to $T$ and $k$ is any positive integer $\geqslant 2$.

Proof: Let $\gamma$ be a geodesic of $M$ passing through $o$. If we put $T=\gamma^{\prime}$, then we have $\gamma^{\prime \prime}=h(T, T)$ and $\gamma^{\prime \prime \prime}=-A_{h(T, T)} T+(\bar{\nabla} h)\left(T^{3}\right)$. Since $\gamma$ is a normal section along $\gamma$,

$$
A_{h(T, T)} T \wedge T=0
$$

Thus, we may put

$$
\gamma^{\prime \prime \prime}=\kappa_{1}^{2} T+(\bar{\nabla} h)\left(T^{3}\right)
$$


where $\kappa_{1}$ is the first curvature of $\gamma$ in $\mathbb{E}^{m}$ given by $\langle h(T, T), h(T, T)\rangle=\kappa_{1}^{2}$. Differentiating $\gamma^{\prime \prime \prime}$ along $\gamma$ gives

$$
\gamma^{(4)}=-\left(T \kappa_{1}^{2}\right) T-\kappa_{1}^{2} h(T, T)-A_{(\bar{\nabla} h)\left(T^{3}\right)} T+\left(\bar{\nabla}^{2} h\right)\left(T^{4}\right),
$$

which gives

$$
\left\langle(\bar{\nabla} h)\left(T^{3}\right), h\left(T, T^{\perp}\right)\right\rangle=0
$$

where $T^{\perp}$ denotes the vector field along $\gamma$ orthogonal to $T$. Similarly, we see that $A_{\left(\bar{\nabla}^{(k-2)} h\right)\left(T^{k}\right)} \wedge T=0$ which implies

$$
\left\langle\left(\bar{\nabla}^{(k-2)} h\right)\left(T^{k}\right), h\left(T, T^{\perp}\right)\right\rangle=0
$$

for all vector field $T^{\perp}$ orthogonal to $\mathrm{T}$, where $k$ is a positive integer $\geqslant 2$.

LEMMA 3.3. Under the same assumption as is stated in Lemma 3.2, we have

$$
\left\langle\left(\bar{\nabla}^{(k-2)} h\right)\left(T^{k}\right),\left(\bar{\nabla}^{(l-2)} h\right)\left(T^{(l-1)}, T^{\perp}\right)\right\rangle=0
$$

for a geodesic $\gamma$ of $M$ passing through $o$ and all integers $k, l \geqslant 2$, where $T=\gamma^{\prime}$ and $T^{\perp}$ is a vector field on $\gamma$ orthogonal to $T$.

Proof: By Lemma 3.2, we see that $\left\langle\left(\bar{\nabla}^{(k-2)} h\right)\left(T^{k}\right), h\left(T, T^{\perp}\right)\right\rangle=0$ holds for a geodesic $\gamma$ passing through $o$, where $T(s)=\gamma^{\prime}(s)$ and $k$ is any positive integer $\geqslant 2$. We shall prove it by the mathematical induction on $l \geqslant 2$. Differentiating the above equation along $\gamma$, we have

$$
\begin{aligned}
0=T\left\langle\left(\bar{\nabla}^{(k-2)} h\right)\left(T^{k}\right), h\left(T, T^{\perp}\right)\right\rangle & \\
=\left\langle\left(\bar{\nabla}^{(k-1)} h\right)\left(T^{(k+1)}\right), h\left(T, T^{\perp}\right)\right\rangle & +\left\langle\left(\bar{\nabla}^{(k-2)} h\right)\left(T^{k}\right),(\bar{\nabla} h)\left(T^{2}, T^{\perp}\right)\right\rangle \\
& +\left\langle\left(\bar{\nabla}^{(k-2)} h\right)\left(T^{k}\right), h\left(T, \nabla_{T^{\perp}} T^{\perp}\right)\right\rangle .
\end{aligned}
$$

Applying Lemma 3.2, we see that the first and third terms vanish. Thus, we get

$$
\left\langle\left(\bar{\nabla}^{(k-2)} h\right)\left(T^{k}\right),(\bar{\nabla} h)\left(T^{2}, T^{\perp}\right)\right\rangle=0 .
$$

for all $k \geqslant 2$.

We now suppose $\left\langle\left(\bar{\nabla}^{(k-2)} h\right)\left(T^{k}\right),\left(\bar{\nabla}^{p} h\right)\left(T^{(p+1)}, T^{\perp}\right)\right\rangle=0$ for $3 \leqslant p \leqslant l-2$ and all $k \geqslant 2$. Then we have

$$
\begin{aligned}
& 0= T\left\langle\left(\bar{\nabla}^{(k-2)} h\right)\left(T^{k}\right),\left(\bar{\nabla}^{p} h\right)\left(T^{(p+1)}, T^{\perp}\right)\right\rangle \\
&=\left\langle\left(\bar{\nabla}^{(k-1)} h\right)\left(T^{(k+1)}\right),\left(\bar{\nabla}^{p} h\right)\left(T^{(p+1)}, T^{\perp}\right)\right\rangle+\left\langle\left(\bar{\nabla}^{(k-2)} h\right)\left(T^{k}\right),\left(\bar{\nabla}^{(p+1)} h\right)\left(T^{p+2}, T^{\perp}\right)\right\rangle \\
&+\left\langle\left(\bar{\nabla}^{(k-2)} h\right)\left(T^{k}\right),\left(\bar{\nabla}^{p} h\right)\left(T^{(p+1)}, \nabla_{T} T^{\perp}\right)\right\rangle .
\end{aligned}
$$

Applying Lemma 3.2 and the induction assumption, we have $\left\langle\left(\bar{\nabla}^{(k-2)} h\right)\left(T^{k}\right),\left(\bar{\nabla}^{(p+1)} h\right)\right.$ $\left.\left(T^{p+2}, T^{\perp}\right)\right\rangle=0$. Thus, Lemma 3.3 is proved. 
Let $\gamma$ be a geodesic of $M$ of order $d$ passing through $o$ in $\mathbb{E}^{m}$ and $\gamma^{\prime}=T$. Since the curvatures $\kappa_{1}, \kappa_{2}, \cdots, \kappa_{d}$ of $\gamma$ can be expressed in terms of $h(T, T) ;(\bar{\nabla} h)\left(T^{3}\right), \ldots$, $\left(\bar{\nabla}^{(k-2)} h\right)\left(T^{k}\right)$ for some positive integer $k$, Lemma 3.2 and 3.3 imply

Proposition 3.4. Let $M$ be a complete submanifold of a Euclidean space $\mathbb{E}^{m}$ with pointed geodesic normal sections at $o$. Then, the curvatures of every geodesic $\gamma$ passing through $o$ are independent of the choice of geodesic. They depend only on the arc length.

Now, we are ready to prove our main theorem.

Let $M$ be a complete submanifold of a Euclidean space $\mathbb{E}^{m}$ with pointed geodesic normal sections at $o$. Choose a geodesic $\gamma$ of $M$ passing through $o$ and let $\gamma^{\prime}=T$. Suppose $\gamma^{\prime} \wedge \gamma^{\prime \prime} \wedge \cdots \wedge \gamma^{(k)} \neq 0$ and $\gamma^{\prime} \wedge \gamma^{\prime \prime} \wedge \cdots \wedge \gamma^{(k+1)}=0$, that is, $\gamma$ is of order $k$ in $\mathbb{E}^{m}$. Then, the geodesics passing through $o$ near by $\gamma$ are of order $k(\geqslant 1)$ by continuity. Therefore, if we consider the geodesic polar coordinate system $\left(s, \theta_{1}, \theta_{2}, \ldots, \theta_{n-1}\right)$ about the point $o$, then we may express $o=x\left(0, \theta_{1}, \theta_{2}, \ldots, \theta_{n-1}\right)$ for all $\theta_{1}, \theta_{2}, \ldots, \theta_{n-1}$ and $\gamma$ as $x\left(s, \bar{\theta}_{1}, \bar{\theta}_{2}, \ldots, \bar{\theta}_{n-1}\right)$ for some fixed $\left(\bar{\theta}_{1}, \bar{\theta}_{2}, \ldots, \bar{\theta}_{n-1}\right)$, where $x$ is the isometric immersion of $M$ into $\mathbb{E}^{m}$. Since all geodesics passing through $o$ near by $\gamma$ are of order $k,\left(\frac{\partial x}{\partial s} \wedge \frac{\partial^{2} x}{\partial s^{2}} \wedge \cdots \wedge \frac{\partial^{k} x}{\partial s^{k}}\right)\left(s, \theta_{i}\right) \neq 0$ for all $\theta_{1}, \ldots, \theta_{n-1} \in \prod_{i=1}^{n-1}\left(\alpha_{i}, \beta_{i}\right)$ containing $\left(\bar{\theta}_{1}, \bar{\theta}_{2}, \ldots, \bar{\theta}_{n-1}\right)$ for some $\alpha_{i}, \beta_{i} \in \mathbb{R}$ such that $\left(\alpha_{i}, \beta_{i}\right)$ is the connected component of $\operatorname{dom} \theta_{i}(i=1, \ldots, n-1)$. Let $\Theta=\prod_{i=1}^{n-1}\left(\alpha_{i}, \beta_{i}\right)$. Suppose there is a limit point $\left(\widetilde{\theta}_{1}, \tilde{\theta}_{2}, \ldots, \tilde{\theta}_{n-1}\right)$ of $\Theta$ such that $\left(\frac{\partial x}{\partial s} \wedge \frac{\partial^{2} x}{\partial s^{2}} \wedge \cdots \wedge \frac{\partial^{k} x}{\partial s^{k}}\right)\left(s_{0}, \tilde{\theta}_{1}, \tilde{\theta}_{2}, \ldots, \tilde{\theta}_{n-1}\right)=0$ for some $s_{0}$. Without loss of generality, we may assume the $(k-1)$-st curvature $\kappa_{k-1}$ of the geodesic $x\left(s, \tilde{\theta}_{1}, \tilde{\theta}_{2}, \ldots, \tilde{\theta}_{n-1}\right)$ vanishes at $\left(s_{0}, \tilde{\theta}_{1}, \tilde{\theta}_{2}, \ldots, \widetilde{\theta}_{n-1}\right)$. By Proposition 3.4 , the Frenet curvatures are independent of the choice of direction, which depend only on the parameter $s$. Thus, $\kappa_{k-1}\left(s_{0}, \theta_{1}, \theta_{2}, \ldots, \theta_{n-1}\right)=0$ for some $\left(\theta_{1}, \theta_{2}, \ldots, \theta_{n-1}\right) \in \Theta$, which is a contradiction. Therefore, the subset $\theta$ is closed and thus the Frenet curvatures of the geodesics passing through $o$ depend not on the direction but on the parameter $s$ only. Hence, the geodesics passing through $o$ are independent of the choice of the direction at $o$, that is, $M$ is geodesically symmetric with respect to $o$. Since $M$ is complete, $M$ is either a Blaschke manifold at $o$ because the cut locus of $o$ is spherical if it has a cut point or a manifold diffeomorphic to a Euclidean space $\mathbb{E}^{n}$ otherwise. Thus, we have

THEOREM 3.5. Let $M$ be a complete submanifold of a Euclidean space $\mathbb{E}^{m}$ with pointed geodesic normal sections at $o$. Then, $M$ is either a Blaschke manifold at $o$ or a manifold diffeomorphic to $\mathbb{E}^{n}$. In particular, all of geodesics passing through $o$ are congruent.

Corollary 3.6. Let $M$ be a complete submanifold of a Euclidean space $\mathbb{E}^{m}$ with pointed geodesic normal sections at o. If one of geodesics is simple, then $M$ is pointed helical at $o$. 
Suppose that a submanifold $M$ in $\mathbb{E}^{m}$ with pointed geodesic normal sections at $o$ for every point $o$ of $M$, that is, the submanifold has geodesic normal sections in the sense of [3]. If we use Lemma 3.2 and Lemma 3.3, it is not difficult to show that all curvatures of geodesics of $M$ are constant independent of the choice of geodesic. In other words, $M$ is helical or, equivalently $M$ has geodesic normal sections in a Euclidean space $\mathbb{E}^{m}$. Combining the results of $[3,12,15]$, we have the classification theorem

THEOREM 3.7. Let $M$ be a complete submanifold of a Euclidean space with geodesic normal sections. Then, $M$ is either a Blaschke manifold or a totally geodesic submanifold.

\section{REFERENCES}

[1] A. Besse, Manifolds all of whose geodesics are closed (Springer-Veriag, Berlin, Heidelberg, New York, 1978).

[2] B.-Y. Chen, Geometry of submanifolds (Marcel Dekker, New York, 1973).

[3] B.-Y. Chen and P. Verheyen, 'Submanifolds with geodesic normal sections', Math. Ann. 269 (1984), 417-429.

[4] S. Fueki, 'Pointed helical submanifolds of pseudo-Riemannian manifolds', J. Geom. 62 (1998), 129-143.

[5] S.L. Hong, 'Isometric immersions of manifolds with plane geodesics into Euclidean space', J. Differential Geom. 8 (1973), 259-278.

[6] Y.H. Kim, 'Surfaces of Euclidean spaces with planar or helical geodesics through a point', Ann. Mat. Pura. Appl. 164 (1993), 1-35.

[7] Y.H. Kim, 'Pointed helical submanifolds of Euclidean space', J. Geom. 50 (1994), 111-117.

[8] Y.H. Kim, 'Pointed planar geodesic submanifolds in Euclidean space', Vietnam J. Math. 31 (2003), 1-8.

[9] A. Little, 'Manifolds with planar geodesics', J. Differential Geom. 11 (1976), 265-285.

[10] B. O'Neill, 'Isotropic and Kaehler immersions', Canad. J. Math. 17 (1965), 907-915.

[11] K. Sakamoto, 'Planar geodesic immersions', Tôhoku Math. J. 29 (1977), 25-56.

[12] K. Sakamoto, 'Helical immersions into a Euclidean space', Michigan Math. J. 33 (1986), 353-364.

[13] K. Sakamoto, 'Helical immersions into a unit sphere', Math. Ann. 261 (1982), 63-80.

[14] K. Tsukada, 'Helical geodesic immersions of compact rank one symmetric spaces into spheres', Tokyo J. Math. 6 (1983), 267-285.

[15] P. Verheyen, 'Submanifolds with geodesic normal sections are helical', Rend. Sem. Mat., Univ. Politec. Torino 43 (1985), 511-527.

Department of Mathematics

Connam National University

Kwangju 535-737

Korea

e-mail: dosokim@chonnam.chonnam.ac.kr
Department of Mathematics

Kyungpook National University

Taegu 702-701

Korea

e-mail: yhkim@knu.ac.kr 